\title{
A Multichannel Edge-Weighted Centroidal Voronoi Tessellation Algorithm for 3D Super-alloy Image Segmentation
}

\author{
Yu Cao ${ }^{1}$, Lili Ju${ }^{2 *}$, Qin Zou ${ }^{1,3}$, Chengzhang $\mathrm{Qu}^{1,4}$, Song Wang ${ }^{1}$ \\ ${ }^{1}$ Department of Computer Science \& Engineering, University of South Carolina, Columbia, SC 29208 \\ ${ }^{2}$ Department of Mathematics, University of South Carolina, Columbia, SC 29208 \\ ${ }^{3}$ School of Remote Sensing \& Infomation Engineering, Wuhan University, Wuhan, China \\ ${ }^{4}$ School of Computer Science, Wuhan University, Wuhan, China \\ cao@cec.sc.edu, ju@math.sc.edu, QZou@live.com, quchengzhang@gmail.com, songwang@cec.sc.edu
}

\begin{abstract}
In material science and engineering, the grain structure inside a super-alloy sample determines its mechanical and physical properties. In this paper, we develop a new Multichannel Edge-Weighted Centroidal Voronoi Tessellation (MCEWCVT) algorithm to automatically segment all the $3 D$ grains from microscopic images of a super-alloy sample. Built upon the classical k-means/CVT algorithm, the proposed algorithm considers both the voxel-intensity similarity within each cluster and the compactness of each cluster. In addition, the same slice of a super-alloy sample can produce multiple images with different grain appearances using different settings of the microscope. We call this multichannel imaging and in this paper, we further adapt the proposed segmentation algorithm to handle such multichannel images to achieve higher grain-segmentation accuracy. We test the proposed MCEWCVT algorithm on a 4-channel Nibased $3 D$ super-alloy image consisting of 170 slices. The segmentation performance is evaluated against the manually annotated ground-truth segmentation and quantitatively compared with other six image segmentation/edgedetection methods. The experimental results demonstrate the higher accuracy of the proposed algorithm than the comparison methods.
\end{abstract}

\section{Introduction}

Super-alloys have been playing a very important role in modern industry. Different applications may require superalloys to have different mechanical or physical properties, such as lightness, hardness, stiffness, electrical conductivity and fluid permeability [20]. In material science, a new super-alloy material is usually designed and developed

\footnotetext{
${ }^{*}$ Corresponding author.
}

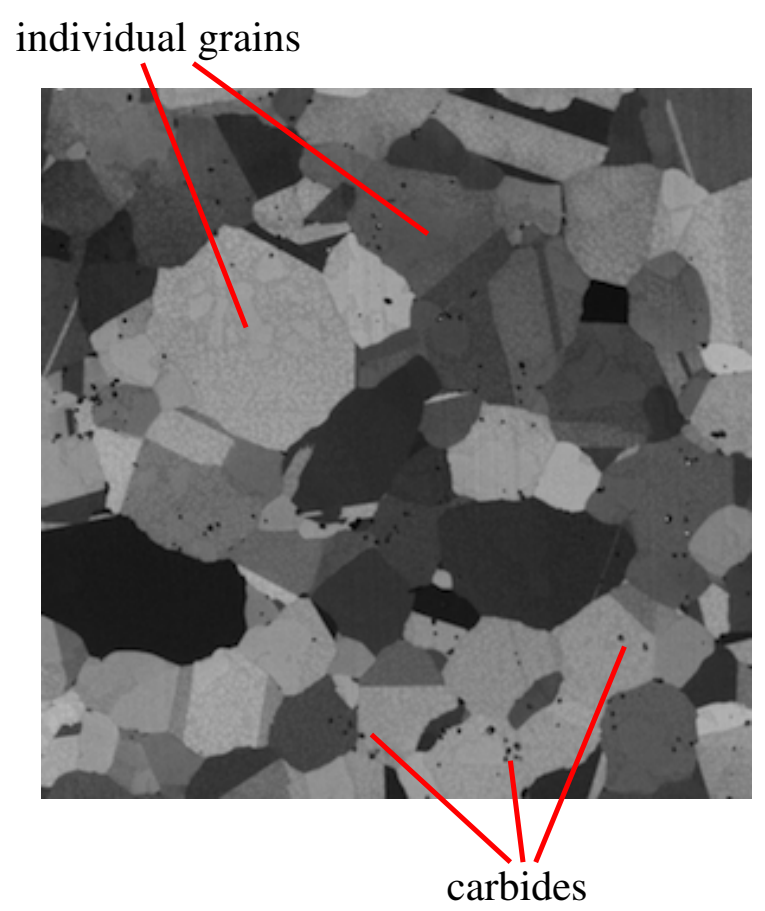

Figure 1. Micro structures in a super-alloy slice.

through repetitive experiments so that the relations between its micro-structures and its mechanical/physical properties can be well identified. Thus, it is critical to identify a superalloy's micro-structure, which is usually in the form of a set of grains [24]. Figure 1 shows an example of the microscopic super-alloy slice image in which cells represent grains and small black points are carbides.

A special and important phenomenon for microscopic super-alloy imaging is that two neighboring grains may be visually separable (e.g., showing different intensities) using one setting of the microscope, but visually inseparable 


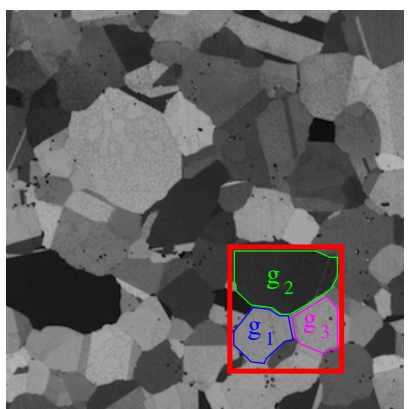

(a)

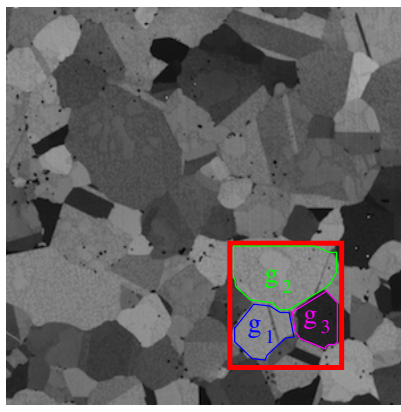

(c)

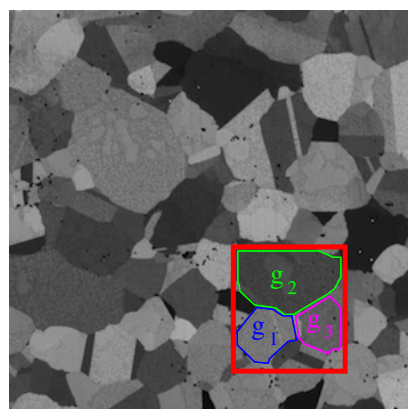

(b)

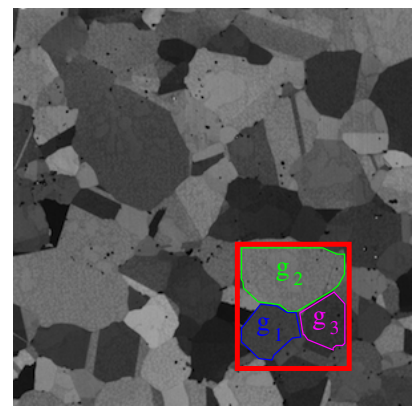

(d)
Figure 2. Microscopic images of a same slice of super-alloy sample taken by using 4 different microscope settings. (a) 4000_Series. (b) 5000_Series. (c) 6000_Series. (d) 7000_Series.

(e.g., showing similar intensities) using another setting of the microscope. In this paper, we use a channel to denote the images produced by a specific setting of the microscope. As an example, Figure 2 shows a 4-channel imaging of the same super-alloy slice. Clearly, it is easier to distinguish grains $g_{1}$ and $g_{2}$ in Fig. 2(a)(b)(d) than in Fig. 2(c). However, it is easier to distinguish grains $g_{1}$ and $g_{3}$ in Fig. 2(c) than in Fig. 2(a)(b)(d). Therefore, the image information in different channels can compensate each other and by combining multichannel microscopic images, we may achieve a more accurate grain segmentation than only using a singlechannel image.

Currently, material scientists have to conduct manual annotation on each 2D slice to segment super-alloy images to obtain the underlying grain structure. The correspondence of the segmented grains in neighboring slices is then identified to reconstruct the 3D grains. Given the large number of grains in a super-alloy sample and the large number of slices in a high-resolution microscopic volume image, manual segmentation is very tedious, time consuming and prone to error. In principle, any existing non-interactive 2D image segmentation method can be used to automate the 2D slice segmentation, such as mean shift [3], watershed [16], statistical region merging [17], normalized cuts [21], graph cuts [10], level set [13] and watershed cuts [4, 5]. However, one major limitation of these $2 \mathrm{D}$ image segmentation meth- ods is that they are operated on each slice independently and do not consider the grain-structure continuity between neighboring slices. Recently, Chuang et al. [2] combined a region merging segmentation method called the stabilized inverse diffusion equation and a stochastic segmentation method called the expectation-maximization/maximization of the posterior marginals (EM/MPM) algorithm for Nibased super-alloy image segmentation. This algorithm is a $2 \mathrm{D}$ segmentation algorithm and is tested only on a single 2D slice.

Direct 3D volume image segmentation has been studied in medical image analysis, such as N-D graph cuts [1], 3D random walks [11], 3D level-set methods [27, 6, 14] and isosurfaces method [25]. These existing 3D segmentation methods may not be suitable for handling the 3D superalloy image segmentation. Particularly, the N-D graph cuts method and the 3D random walks method require manually specified seeds for segmentation, which is also tedious, time consuming and prone to error. Furthermore, both the 3D level-set methods and the isosurface methods assume that there are only a small number of disjoint structures of interest (e.g., the organs, tissues, or tumors in medical imaging applications). In our case, however, a super-alloy sample usually contains hundreds or even thousands of grains that are adjacent to each other. This will substantially increase the model and computational complexity of the level-set and isosurface method and make them even unacceptable and infeasible. In addition, the above-mentioned 2D and $3 \mathrm{D}$ segmentation methods usually do not consider the problem of multichannel image segmentation.

In this paper, we propose a Multichannel Edge-Weighted Centroidal Voronoi Tessellation (MCEWCVT) algorithm for 3D multichannel super-alloy image segmentation. Following the general principle of the k-means/CVT clustering algorithm, the proposed algorithm modifies and generalizes the idea of Edge-Weighted Centroidal Voronoi Tessellation (EWCVT) model developed in [26] in three aspects: 1) an edge detection function is added into the classic clustering energy as a density in the EWCVT model, 2) the 3D boundary continuity constraint is applied by extending edge definition from 2D segments to 3D faces, and 3) the multichannel model is added into the similarity measure for clustering. These three improvements make the proposed algorithm particularly suitable for 3D super-alloy image segmentation.

The remainder of this paper is organized as follows. In Section 2, we start with a brief review of the basic Centroidal Voronoi Tessellation (CVT) model and then derive a multichannel edge-weighted clustering energy for segmenting multichannel 3D super-alloy images. In Section 3, we develop the MCEWCVT model for characterizing the minimizers of the new clustering energy and propose an effective solution algorithm. In Section 4, we test the proposed 
MCEWCVT algorithm on a 3D super-alloy image and evaluate its performance quantitatively. Section 5 concludes the paper.

\section{The Multichannel Edge-Weighted Cluster- ing Energy}

An image of the 3D super-alloy sample can be regarded as a function $u$ defined on a domain $\Omega \subseteq \mathbb{R}^{3}$ where the values of $u$ represent the gray levels (intensity) of the voxels. Furthermore, since the voxels are usually indexed by integer triplets, we can treat $u$ as a discrete function defined over a set of points with integer coordinates, i.e., the point $(x, y, z)=(i, j, k)$, where $(i, j, k)$ is an integer triplet that ranges over the volume domain. Thus, the domain of $u$ for a super-alloy volume is an index set $D=\{(i, j, k) \mid i=$ $1, \ldots, I, j=1, \ldots, J, k=1, \ldots, K\}$ for some positive integers $I, J$ and $K$.

\subsection{Basic centroidal Voronoi tessellation model}

Let $\mathbb{U}=\{u(i, j, k)\}_{(i, j, k) \in D}$ denote the set of intensity values of the $3 \mathrm{D}$ super-alloy image and $\mathcal{W}=\left\{w_{l}\right\}_{l=1}^{L}$ a set of typical intensity levels. The Voronoi region $V_{l}(l=$ $1, \ldots, L)$ in $\mathbb{U}$ corresponding to $w_{l}$ is defined by

$$
\begin{array}{r}
V_{l}=\left\{u(i, j, k) \in \mathbb{U}|| u(i, j, k)-w_{l} \mid<\right. \\
\left.\left|u(i, j, k)-w_{m}\right|, m=1,2, \ldots, L\right\},
\end{array}
$$

where $|\cdot|$ is some predefined metric measure such as the Euclidean distance. The set $\mathcal{V}=\left\{V_{l}\right\}_{l=1}^{L}$ is called a Voronoi tessellation or Voronoi clustering $[18,7,8]$ of the set $\mathbb{U}$. The set of chosen intensities $\mathcal{W}=\left\{w_{l}\right\}_{l=1}^{L}$ are referred as the Voronoi generators. Since we have $V_{i} \bigcap V_{j}=\emptyset$ if $i \neq j$ and $\mathbb{U}=\bigcup_{l=1}^{L} \bar{V}_{l}$, the Voronoi tessellation $\mathcal{V}$ can be viewed as a special partition of $\mathbb{U}$.

Let $\rho$ be a predefined density function defined on $D$. Given a partition of $\mathbb{U}$, denoted by $\left\{U_{l}\right\}_{l=1}^{L}$, the centroid ( i.e., center of mass or cluster mean) of each cell $U_{l}$, with respect to the density $\rho$, is defined to be the intensity $w_{l}^{*} \in U_{l}$ which minimizes

$$
\min _{w \in U_{l}} \sum_{u(i, j, k) \in U_{l}} \rho(i, j, k)|u(i, j, k)-w|^{2}
$$

We note that this centroid definition is slightly different from that proposed in [26] with the newly introduced density function. For an arbitrary Voronoi tessellation $\left(\left\{w_{l}\right\}_{l=1}^{L} ;\left\{V_{l}\right\}_{l=1}^{L}\right)$ of $\mathbb{U}$, it may not always be the case that $w_{l}=w_{l}^{*}$ for $l=1,2, \ldots, L$, where $\left\{w^{*}\right\}_{l=1}^{L}$ are the corresponding centroids of $\left\{V_{l}\right\}_{l=1}^{L}$. If the generators of the Voronoi regions $\left\{V_{l}\right\}_{l=1}^{L}$ of $\mathbb{U}$ coincide with their corresponding centroids, i.e.,

$$
w_{l}=w_{l}^{*}, \quad \text { for } \quad l=1,2, \ldots, L,
$$

then we call the Voronoi tessellation $\left\{V_{l}\right\}_{l=1}^{L}$ a centroidal Voronoi tessellation (CVT) [7] of $\mathbb{U}$ and refer to $\left\{w_{l}\right\}_{l=1}^{L}$ as the corresponding CVT generators.

The construction of CVTs often can be achieved by an "energy" minimization process [7]. Generally, for any set of points $\mathcal{W}=\left\{w_{l}\right\}_{l=1}^{L}$ and any partition $\mathcal{U}=\left\{u_{l}\right\}_{l=1}^{L}$ of $\mathbb{U}$, the classic clustering energy of $(\mathcal{W} ; \mathcal{U})$ can be defined as follows:

$$
E_{C}(\mathcal{W} ; \mathcal{U})=\sum_{l=1}^{L} \sum_{u(i, j, k) \in U_{l}} \rho(i, j, k)\left|u(i, j, k)-w_{l}\right|^{2}
$$

Suppose that we have determined the clusters $\left\{U_{l}\right\}_{l=1}^{L}$ for a given 3D super-alloy image represented by $u(i, j, k)$ for $(i, j, k) \in D$. Then a segmentation in physical space of the image can be naturally produced. Define segmentation in physical space as $\mathcal{D}=\left\{D_{l}\right\}_{l=1}^{L}$, where

$$
D_{l}=\left\{(i, j, k) \mid u(i, j, k) \in U_{l}\right\} .
$$

Consequently, the classic clustering energy (3) can be rewritten in physical segmentation terminology as

$$
E_{C}(\mathcal{W} ; \mathcal{D})=\sum_{l=1}^{L} \sum_{(i, j, k) \in D_{l}} \rho(i, j, k)\left|u(i, j, k)-w_{l}\right|^{2} .
$$

It is well known from [7] that $(\mathcal{W} ; \mathcal{D})$ is a minimizer of $E_{C}(\mathcal{W} ; \mathcal{D})$ only if $(\mathcal{W} ; \mathcal{D})$ forms a CVT of $D$. Several algorithms of minimizing the above energy function can be found in $[12,22,23]$.

\subsection{Multichannel clustering energy}

Let $N$ denote the number of channels, i.e., we have $N$ images, $u^{1}, u^{2}, \cdots, u^{N}$, of the same super-alloy sample taken under different microscope settings. Now we can rewrite $\mathbb{U}$ and $\mathcal{W}$ in the vector form

$$
\mathbb{U}=\left\{\vec{u}(i, j, k)=\left(u^{1}, u^{2}, \cdots, u^{N}\right)^{T} \in \mathbb{R}^{N}\right\}_{(i, j, k) \in D}
$$

and

$$
\mathcal{W}=\left\{\vec{w}_{l}=\left(w_{l}^{1}, w_{l}^{2}, \cdots, w_{l}^{N}\right)^{T} \in \mathbb{R}^{N}\right\}_{l=1}^{L},
$$

respectively.

Note that one grain may be visually separable in one channel but has the close intensity value with its adjacent grains in other channels. Thus, we need to choose proper measurement of the distance for $\vec{u}(i, j, k)$ and $\vec{w}_{l}$ which can effectively capture those grains only distinct from its adjacent grains in some of the channels. We here take the $\infty$-norm which is defined to be

$$
\|\vec{x}\|_{\infty}=\max \left(\left|x^{1}\right|,\left|x^{2}\right|, \ldots,\left|x^{N}\right|\right)
$$

where $\vec{x}=\left(x^{1}, x^{2}, \ldots, x^{N}\right) \in \mathbb{R}^{N}$. 
Thus, we can rewrite the classic clustering (4) as

$$
E_{C}(\mathcal{W} ; \mathcal{D})=\sum_{l=1}^{L} \sum_{(i, j, k) \in D_{l}} \rho(i, j, k)\left\|\vec{u}(i, j, k)-\vec{w}_{l}\right\|_{\infty}^{2} .
$$

Note that (5) will reduce to (4) when $N=1$.

\subsection{Edge energy}

As mentioned before, we would like to apply constraints to enforce the continuity and smoothness on edges (faces) of the 3D super-alloy grains/segments. Now we define an edge related energy term for a given clustering $\mathcal{D}$ of the physical space similar to that proposed in [26].

For each voxel $(i, j, k) \in D$, denote by $\mathbb{N}_{\omega}(i, j, k)$ a local neighborhood for it, which could be a $\omega \times \omega \times \omega$ cube centered at $(i, j, k)$ or a sphere centered at $(i, j, k)$ with ra$\operatorname{dius} \omega$.

We define a local characteristic function $\chi_{(i, j, k)}$ : $\mathbb{N}_{\omega}(i, j, k) \rightarrow\{0,1\}$ as

$\chi_{(i, j, k)}\left(i^{\prime}, j^{\prime}, k^{\prime}\right)= \begin{cases}1 & \text { if } \pi_{u}\left(i^{\prime}, j^{\prime}, k^{\prime}\right) \neq \pi_{u}(i, j, k), \\ 0 & \text { otherwise, }\end{cases}$

where $\pi_{u}(i, j, k): D \rightarrow\{1, \ldots, L\}$ tells which cluster $\vec{u}(i, j, k)$ belongs to. Then an edge energy can be defined as

$$
E_{L}(\mathcal{D})=\sum_{(i, j, k) \in D} \sum_{\left(i^{\prime}, j^{\prime}, k^{\prime}\right) \in \mathbb{N}_{\omega}(i, j, k)} \chi_{(i, j, k)}\left(i^{\prime}, j^{\prime}, k^{\prime}\right) .
$$

Generalizing the analysis for 2D cases in [26], it is not too hard to demonstrate that $E_{L}$ is proportional to $\omega^{4} A$ in the asymptotic sense where $A$ is the area of the boundaries between the 3D segments. Note that the edge energy has nothing to do with the generators $\mathcal{W}$.

\subsection{Total energy}

In order to enforce the detection of edges, we take the density function $\rho$ as

$$
\rho=1+|\nabla \vec{u}|
$$

By combining (5), (6) and (7), we then define the total energy for our model, i,e., the multichannel edge-weighted clustering energy, to be as follows:

$$
\begin{aligned}
& E(\mathcal{W} ; \mathcal{D})=E_{C}(\mathcal{W} ; \mathcal{D})+\lambda E_{L}(\mathcal{D}) \\
& =\sum_{l=1}^{L} \sum_{(i, j, k) \in D_{l}}(1+|\nabla \vec{u}(i, j, k)|)\left\|\vec{u}(i, j, k)-\vec{w}_{l}\right\|_{\infty}^{2} \\
& \quad+\lambda \sum_{(i, j, k) \in D} \sum_{\left(i^{\prime}, j^{\prime}, k^{\prime}\right) \in \mathbb{N}_{\omega}(i, j, k)} \chi_{(i, j, k)}\left(i^{\prime}, j^{\prime}, k^{\prime}\right)
\end{aligned}
$$

where $\lambda$ is a weighting function to control the balance between $E_{C}$ and $E_{L}$.

\section{The Multichannel Edge-Weighted Cen- troidal Voronoi Tessellation Algorithm}

In this section, we introduce the way of minimizing the multichannel edge-weighted clustering energy function (8) based on the CVT-methodology [7, 9].

\subsection{Multichannel edge-weighed distance}

Basically the energy minimization is achieved through the process of transferring voxels from one cluster to another. We want to guarantee that transferring a voxel to the associated cluster of the generator to which it has the shortest multichannel edge-weighted distance decreases the total multichannel edge-weighted clustering energy the most.

Let us rewrite equation (8) as

$$
\begin{aligned}
& E(\mathcal{W} ; \mathcal{D})= \\
& \quad \sum_{\left(i^{\prime}, j^{\prime}, k^{\prime}\right) \in D \backslash(i, j, k)} \rho\left(i^{\prime}, j^{\prime}, k^{\prime}\right)\left\|\vec{u}\left(i^{\prime}, j^{\prime}, k^{\prime}\right)-\vec{w}_{\pi_{u}\left(i^{\prime}, j^{\prime}, k^{\prime}\right)}\right\|_{\infty}^{2} \\
& +\rho(i, j, k)\left\|\vec{u}(i, j, k)-\vec{w}_{\pi_{u}(i, j, k)}\right\|_{\infty}^{2} \\
& +\sum_{\left(i^{\prime}, j^{\prime}, k^{\prime}\right) \in D \backslash(i, j, k)} \epsilon_{\mathcal{L}}\left(i^{\prime}, j^{\prime}, k^{\prime}\right) \\
& +\epsilon_{\mathcal{L}}(i, j, k)
\end{aligned}
$$

where

$$
\epsilon_{\mathcal{L}}(i, j, k)=\lambda \sum_{\left(i^{\prime}, j^{\prime}, k^{\prime}\right) \in \mathbb{N}_{\omega}(i, j, k)} \chi_{(i, j, k)}\left(i^{\prime}, j^{\prime}, k^{\prime}\right) .
$$

Now we consider the variation of the total energy when transferring a voxel $(i, j, k)$ from its current cluster $D_{l}$ to another cluster $D_{m}$. Examining equation (9), the first term on the right side has no change. The change of second term is

$$
\rho(i, j, k)\left(\left\|\vec{u}(i, j, k)-\vec{w}_{m}\right\|_{\infty}^{2}-\left\|\vec{u}(i, j, k)-\vec{w}_{l}\right\|_{\infty}^{2}\right) .
$$

Denote $n_{k}(i, j, k)$ the number of voxels within $\left(D_{k} \bigcap \mathbb{N}_{w}(i, j, k)\right) \backslash(i, j, k)$. By the analysis from [26], we know that the changes in the third and fourth term after transferring are both equal to

$$
\begin{aligned}
& \lambda n_{l}(i, j, k)- \lambda n_{m}(i, j, k)= \\
& \lambda\left[n_{l}(i, j, k)-n_{m}(i, j, k)\right]
\end{aligned}
$$

Summarizing (10) and (11), we have the overall variation of the total energy by transferring voxel $(i, j, k)$ from cluster $D_{l}$ to $D_{m}$ as

$$
\begin{gathered}
\rho(i, j, k)\left(\left\|\vec{u}(i, j, k)-\vec{w}_{m}\right\|_{\infty}^{2}-\left\|\vec{u}(i, j, k)-\vec{w}_{l}\right\|_{\infty}^{2}\right)+ \\
2 \lambda\left(n_{l}(i, j, k)-n_{m}(i, j, k)\right)
\end{gathered}
$$

which can be rewritten as

$$
\begin{aligned}
& {\left[\rho(i, j, k)\left\|\vec{u}(i, j, k)-\vec{w}_{m}\right\|_{\infty}^{2}-2 \lambda n_{m}(i, j, k)\right]} \\
& \quad-\left[\rho(i, j, k)\left\|\vec{u}(i, j, k)-\vec{w}_{l}\right\|_{\infty}^{2}-2 \lambda n_{l}(i, j, k)\right] .
\end{aligned}
$$


Thus we define the multichannel edge-weighted distance from a voxel $(i, j, k)$ to generator $w_{k}$ to be

$$
\begin{aligned}
& \operatorname{dist}\left((i, j, k), \vec{w}_{k}\right) \\
& =\sqrt{\rho(i, j, k)\left\|\vec{u}(i, j, k)-\vec{w}_{k}\right\|_{\infty}^{2}+2 \lambda \tilde{n}_{k}(i, j, k)} \\
& =\sqrt{(1+|\nabla \vec{u}(i, j, k)|)\left\|\vec{u}(i, j, k)-\vec{w}_{k}\right\|_{\infty}^{2}+2 \lambda \tilde{n}_{k}(i, j, k)}
\end{aligned}
$$

where $\tilde{n}_{k}(i, j, k)=\left|\mathbb{N}_{w}(i, j, k)\right|-n_{k}(i, j, k)-1$ is the number of voxels in $\mathbb{N}_{w}(i, j, k) \backslash\left(D_{k} \bigcup(i, j, k)\right)$.

In conclusion, moving a voxel to the cluster of a generator to which it has the shortest edge-weighted distance defined by (13) will decrease the total clustering energy $E(\mathcal{W} ; \mathcal{D})$ the most.

\subsection{Multichannel edge-weighted Voronoi regions}

Given a set of multichannel generators $\mathcal{W}=\left\{\vec{w}_{l}\right\}_{l=1}^{L}$, we can define the multichannel edge-weighted Voronoi region $\tilde{\mathcal{D}}=\left\{\tilde{D}_{l}\right\}_{l=1}^{L}$ in the physical volume space $D$ as

$$
\begin{gathered}
\tilde{D}_{l}=\left\{(i, j, k) \in D \mid \operatorname{dist}\left((i, j, k), \vec{w}_{l}\right) \leq \operatorname{dist}\left((i, j, k), \vec{w}_{m}\right),\right. \\
m=1, \ldots, L\} .
\end{gathered}
$$

From equations (12) and (13), it is easy to find that when $\mathcal{W}$ is fixed, the multichannel edge-weighted Voronoi tessellation $\tilde{\mathcal{D}}=\left\{\tilde{D}_{l}\right\}_{l=1}^{L}$ associated with $\mathcal{W}$ corresponds to the minimizer of the multichannel edge-weighted energy $E(\mathcal{W} ; \mathcal{D})$, i.e.,

$$
\tilde{\mathcal{D}}=\arg \min _{\mathcal{D}} E(\mathcal{W} ; \mathcal{D})
$$

Then we define the multichannel edge-weighted Voronoi tessellation energy for a given set of generators $\mathcal{W}=$ $\left\{w_{l}\right\}_{l=1}^{L}$ to be

$$
E_{M C E W V T}(\mathcal{W})=E(\mathcal{W} ; \tilde{\mathcal{D}}) .
$$

Algorithm 1 can be used to efficiently construct the multichannel edge-weighed Voronoi regions for a given set of generators.

\subsection{The MCEWCVT model and its implementation}

In order to define the MCEWCVT model, we need to further determine the centroids of a given set of partition $\tilde{\mathcal{D}}=\left\{\tilde{D}_{l}\right\}_{l=1}^{L}$ of $D$, i.e., find $\mathcal{W}=\left\{\vec{w}_{l}^{*}\right\}_{l=1}^{L}$ such that

$$
\vec{w}_{l}^{*}=\arg \min _{\vec{w}} \sum_{(i, j, k) \in \tilde{D}_{l}} \rho(i, j, k)\|\vec{u}(i, j, k)-\vec{w}\|_{\infty}^{2}
$$

for $l=1,2, \cdots, L$. Since we use the $\infty$-norm, it is hard to find an analytical solution for $\vec{w}_{l}$. Usually, the $\vec{w}_{l}$ defined through the above minimization process could be solved numerically. For example, the Powell method [19] could be used to effectively calculate $\vec{w}_{l}$ approximately although there is no derivative information available.

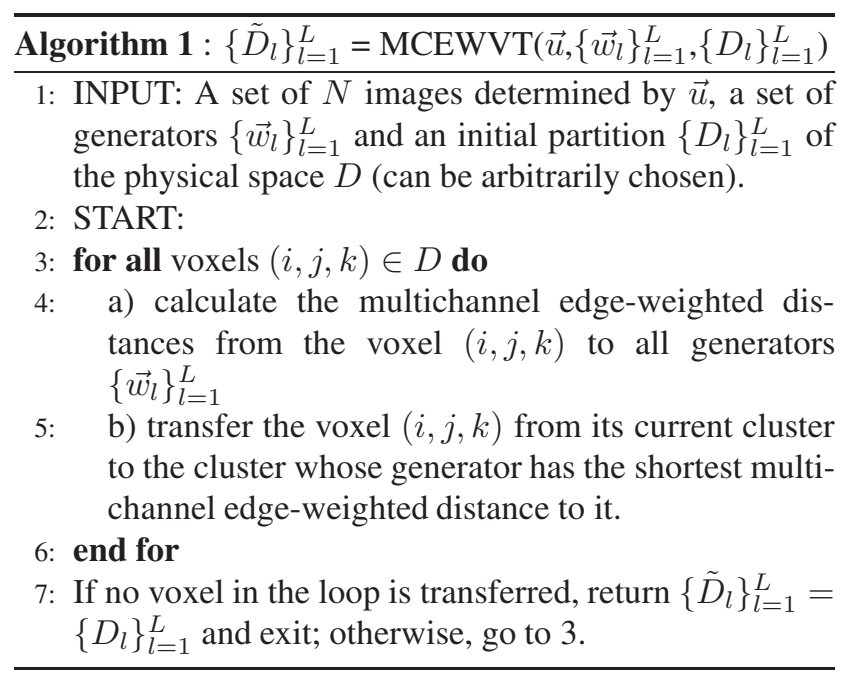

Definition (MCEWCVT) For a given multichannel edgeweighted Voronoi tessellation $\left(\left\{\vec{w}_{l}\right\}_{l=1}^{L} ;\left\{\tilde{D}_{l}\right\}_{l=1}^{L}\right)$ of $D$, we call it a multichannel edge-weighted centroidal Voronoi tessellation (MCEWCVT) of D if the generators $\left\{\vec{w}_{l}\right\}_{l=1}^{L}$ are also the corresponding centroids of the associated multichannel edge-weighted Voronoi regions $\left\{\tilde{D}_{l}\right\}_{l=1}^{L}$, i.e.,

$$
\vec{w}_{l}=\vec{w}_{l}^{*}, \quad l=1,2, \cdots, L .
$$

Based on the CVT principle, we know that $(\mathcal{W} ; \tilde{\mathcal{D}})$ is a minimizer of $E(\mathcal{W} ; \tilde{\mathcal{D}})$ only if $(\mathcal{W} ; \tilde{\mathcal{D}})$ forms a MCEWCVT of $D$. We propose the Algorithm 2 for construction of the MCEWCVTs. As discussed in [26] for the EWCVT construction algorithms, some improvements of Algorithm 2-MCEWCVT can be obtained by using narrowbanded implementation and better initialization processes. We also note that the energy $E_{M C E W V T}(\mathcal{W})$ keeps decreasing along the iterations in this algorithm.

Algorithm 2: $\left(\left\{\vec{w}_{l}\right\}_{l=1}^{L},\left\{\tilde{D}_{l}\right\}_{l=1}^{L}\right)=\operatorname{MCEWCVT}(\vec{u}, L)$

1: INPUT: A set of $N$ images determined by $\vec{u}$ and an integer $L$.

2: START:

3: Arbitrarily choose an initial partition $\left\{\tilde{D}_{l}\right\}_{l=1}^{L}$ of the physical space $D$ or by some means.

4: For each cluster $\tilde{D}_{l}, l=1, \ldots, L$, calculate its cluster centroid $\vec{w}_{l}^{*}$.

5: Take $\left\{\vec{w}_{l}^{*}\right\}_{l=1}^{L}$ as the generators, determine the corresponding multichannel edge-weighted Voronoi clustering $\left\{\tilde{D}_{l}^{\prime}\right\}_{l=1}^{L}$ by using Algorithm 1-MCEWVT, i.e., $\left\{\tilde{D}_{l}^{\prime}\right\}_{l=1}^{L}=\operatorname{MCEWVT}\left(\vec{u},\left\{\vec{w}_{l}^{*}\right\}_{l=1}^{L},\left\{\tilde{D}_{l}\right\}_{l=1}^{L}\right)$.

6: If $\left\{\tilde{D}_{l}^{\prime}\right\}_{l=1}^{L}$ and $\left\{\tilde{D}_{l}\right\}_{l=1}^{L}$ are the same, return $\left(\left\{\vec{w}_{l}\right\}_{l=1}^{L} ;\left\{\tilde{D}_{l}\right\}_{l=1}^{L}\right)$ and exit; otherwise, set $\tilde{D}_{l}=\tilde{D}_{l}^{\prime}$ for $l=1, \ldots, L$ and go to step 4 . 


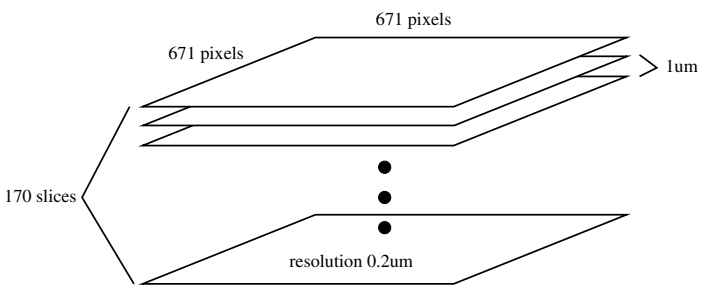

Figure 3. An illustration of the super-alloy volume images.

\section{Experiments}

In this section, we start with introducing more details of the testing super-alloy image dataset. Then we discuss the parameter settings for the MCEWCVT algorithm. At last, we present the visual and quantitative comparison of the MCEWCVT algorithm with other six image segmentation/edge-detection methods.

The proposed segmentation method was tested on IN100 Ni-based super-alloy dataset provided by Prof. Marc De Graef from Carnegie Mellon University. The dataset consists 4 channels of super-alloy slice images which were taken using different microscope settings. Each slice image was photographed as new facets appearing by keeping abrading the up-front facet of the super-alloy sample. The size of the slice image is $671 \times 671$ and the number of slice images in each channel is 170 . The physical distance between two neighbor voxels on the slice image is $0.2 \mu \mathrm{m}$ and that between two adjacent slices is $1 \mu \mathrm{m}$. Figure 3 illustrates the informations of the super-alloy sample.

From Fig. 3, we can notice that the physical distance between two adjacent slices is about 5 times of the physical distance between two neighbor voxel on a single slice. The MCEWCVT segmentation algorithm expects the sampling rate of the 3D super-alloy image on each axis to be the same so that the smoothness and compactness of the edge of segmented grains can be well controlled. Thus, we interpolate the original 3D super-alloy image with 4 more slices between each two original slices by linear combination of those two original slices. Then the interpolated super-alloy volume image has 846 slices. We tested the MCEWCVT algorithm on the interpolated $3 \mathrm{D}$ image, which can produce a 3D segmentation result.

There are 4 parameters of the MCEWCVT algorithm that can be tuned during the experiments. $L$, the number of clusters (note that it is not the number of grained contained by the super-alloy volume); $\lambda$, the weighting parameter which balances the multichannel clustering energy term and the edge energy term; $\omega$, the neighbor size which defines the local search region $\mathbb{N}_{\omega}(i, j, k)$ for each voxel $(i, j, k) . \epsilon$, the predefined threshold for the stop condition of MCEWCVT. In the experiments, we let $\mathbb{N}_{\omega}(i, j, k)$ be a sphere centered at voxel $(i, j, k)$ and choose $L=30$,

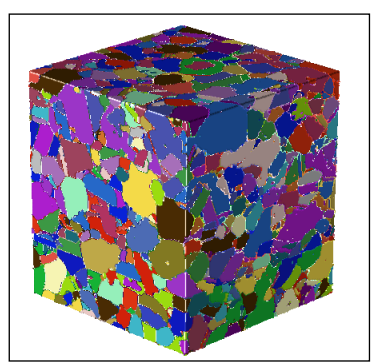

(a)

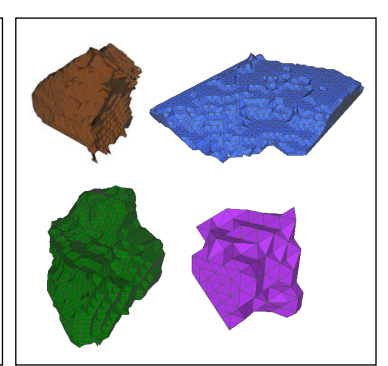

(b)
Figure 4. (a) Visualization of the 3D segmentation results. Segments are represented with different colors. (b) Segmented grains.

$\lambda=300, \omega=6$ considering the size of carbides and energy balance, see discussions in [26]. Theoretically, Algorithm 2-MCEWCVT stops when the energy function (8) is completely minimized. However, in practical applications we may set the stop condition as

$$
\frac{\left|E_{i+1}-E_{i}\right|}{E_{i}}<\epsilon
$$

where $E_{i}$ denotes the MCEWVT energy at the $i$-th iteration and $\epsilon$ is a predefined threshold. In the experiments, we selected $\epsilon=10^{-4}$.

In the experiment, we used the basic CVT clustering results as the initial partition. And it took 6 iterations and about 10 hours for the MCEWCVT algorithm to converge when running on a Dell T7400 workstation using one out of 8 cores $(2.0 \mathrm{GHz})$ and 4 out of $8 \mathrm{~GB}$ memory. Figure 4 shows a visualization of the segmentation results on the volume and segmented grain samples.

To quantitatively evaluate the accuracy and performance of the MCEWCVT algorithm, we calculated its boundary accuracy against the manually annotated groundtruth segmentation and compared the boundary accuracy with other six image segmentation/edge-detection methods. We constructed the segmentation ground-truth on the uninterpolated 170 slices by manually labeling the grains' boundaries on each 2D slice, which is same as what has to be done in current material researches. Thus, we need the MCEWCVT and the comparison methods can provide 2D segmentation results for comparing against the groundtruth. For the MCEWCVT algorithm, we first project the $3 \mathrm{D}$ boundary results onto 846 slices, then we remove those slices which produced by interpolations. And then we take the boundary results on the rest 170 slices into the comparison. As for the comparison methods, in order to follow the preference of super-alloy application (i.e., prefer no human interactions), we choose automatic non-interactive 2D segmentation/edge-detection methods including meanshift [3], srm [17], pbCanny edge detector [15], pbCGTG edge detector [15], gpb [28] and watershed [16]. And 


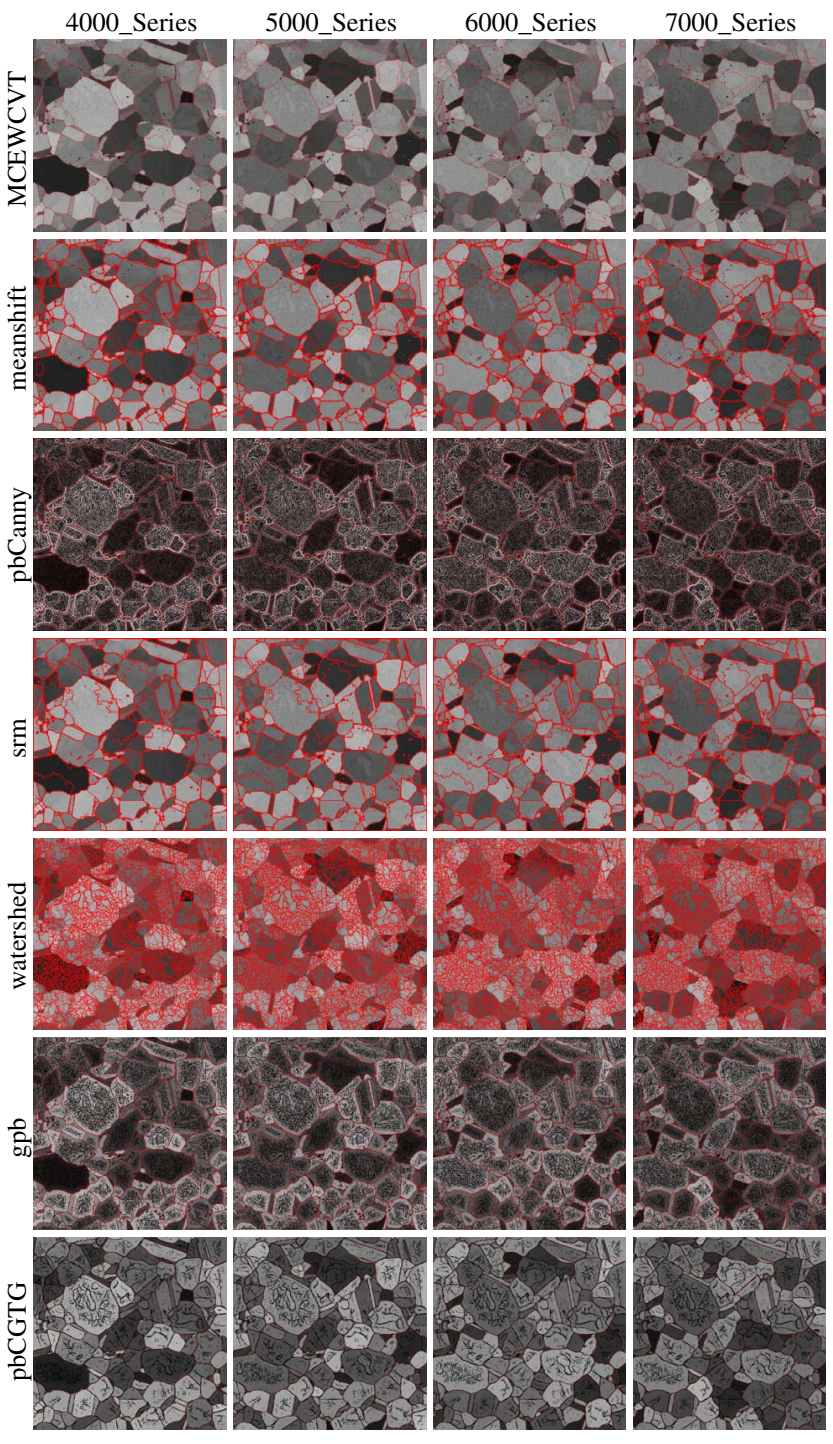

Figure 5. Visual comparisons (one slice) of the segmentation results of the proposed MCEWCVT algorithm and six comparison methods.

the visual comparison is given in Fig. 5. As we can see, the MCEWCVT segmentation results more accurately and clearly delineate the grains' boundaries than the comparison segmentation results.

From Fig. 5, we can notice that the MCEWCVT can produce an unique segmentation result for all 4 channel images, while the 2D comparison methods will produce different segmentation results for different channel images due to their incapability of handling multichannel image segmentation. Thus, for comparison methods, we combine the segmentations in all 4 channels to obtain an unique segmentation for a single slice. Specificity, for pb edge detectors, we assign the $\mathrm{pb}$ value for each pixel $(i, j)$ with the maximum $\mathrm{pb}$ value from all 4 channels at the pixel $(i, j)$. For the rest of the comparison methods, we combine the segmentations from all 4 channels through logic OR operations.

For the quantitative comparison, we use segmentation evaluation codes provided in the Berkeley segmentation benchmark [15] to calculate the precision-recall curve and $F$-measure(harmonic mean of the precision and recall).

Figure 6 shows the quantitative comparisons on segmentation results of the MCEWCVT algorithm and its comparison methods. For pb edge detectors, doing thresholding

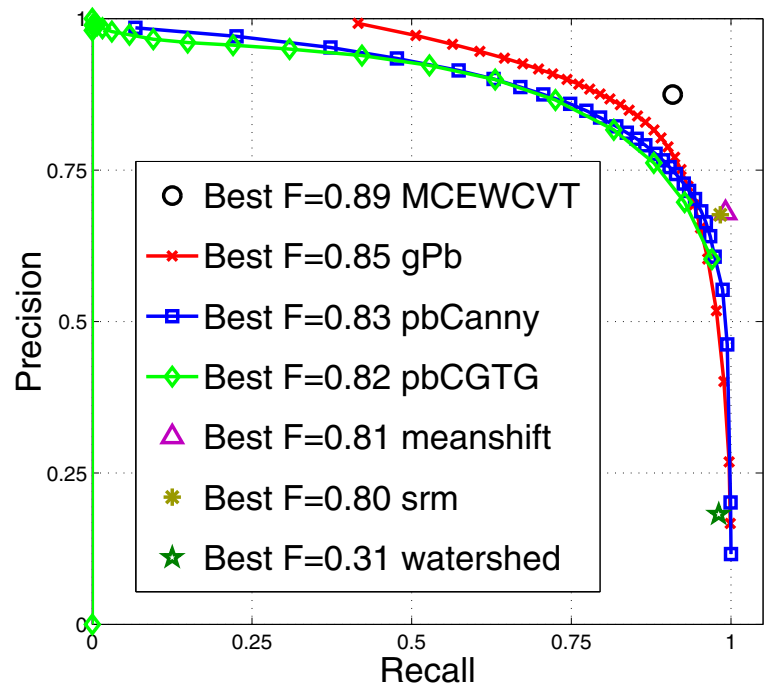

Figure 6. Quantitative comparisons between the MCEWCVT algorithm and its comparison methods.

of the gray intensity from 0 to 255 on a boundary map can produce a series of binarized solid boundary images. Then the precision-recall curve can be calculated based on these boundary images. Subsequently, $F$-measure for each point on the precision-recall curve can be calculated using

$$
F=2 \cdot \frac{\text { precision } \cdot \text { recall }}{\text { precision }+ \text { recall }}
$$

For the rest comparison methods, solid segmentation boundaries correspond to a single precision-recall value. Thus, we compare the proposed MCEWCVT algorithm with its comparison methods on their best $F$ measures. Generally, the higher best $F$-measure, the better the segmentation/edge-detection performance is. From Fig. 6, we can see that the proposed MCEWCVT algorithm achieves a best $F$-measure of 0.89 which outperforms the six comparison 2D segmentation/edge-detection methods.

\section{Conclusions}

In this paper, we developed a multichannel edgeweighted centroidal Voronoi tessellations algorithm for 3D 
microscopic super-alloy image segmentation. As a volumetric segmentation algorithm, it can automatically extract the 3D grains of a super-alloy sample, without any human interaction. Compared to previous 2D EWCVT method, the proposed algorithm is able to handle 3D multichannel super-alloy images that can substantially increase the segmentation performance. We conducted experiments on a 3D Ni-based super-alloy image that consists of 170 4-channel super-alloy slices and the visual and quantitative comparison results indicate that the performance of the proposed algorithm is better than other six existing image segmentation/edge-detection methods for this task.

\section{Acknowledgments}

We would like to thank Professor Marc De Graef from Carnegie Mellon University for providing the super-alloy images. This work was supported, in part, by NSF DMS0913491, NSF IIS-0951754, NSF IIS-1017199 and Army Research Laboratory under Cooperative Agreement Number W911NF-10-2-0060. The views and conclusions contained in this document are those of the authors and should not be interpreted as representing the official policies, either express or implied, of the Army Research Laboratory or the U.S. Government. The U.S. Government is authorized to reproduce and distribute reprints for Government purposes, notwithstanding any copyright notation herein.

\section{References}

[1] Y. Boykov and G. Funka-Lea. Graph cuts and efficient N-D image segmentation. IJCV, Vol. 70, pp. 109-131, 2006.

[2] H.C. Chuang, L.M. Huffman, M.L. Comer, J.P. Simmons, and I. Pollak. An automated segmentation for nickel-based superalloy. In IEEE ICIP, pp. 2280-2283, 2008.

[3] D. Comaniciu, P. Meer, and S. Member. Mean shift: A robust approach toward feature space analysis. IEEE PAMI, Vol. 24, pp. 603-619, 2002.

[4] C. Couprie, L. Grady, L. Najman, and H. Talbot. Power watersheds: A new image segmentation framework extending graph cuts, random walker and optimal spanning forest. ICCV , pp. 731-738, 2009.

[5] J. Cousty, G. Bertrand, L. Najman and M. Couprie. Watershed cuts: thinnings, shortest-path forests and topological watersheds IEEE PAMI, Vol. 32, pp. 925-939, 2010.

[6] K.M. David, D.E. Breen, L. Zhukov, and R.T. Whitakery. Level set segmentation from multiple non-uniform volume datasets. In IEEE Conf. Visualization, pp. 179-186, 2002.

[7] Q. Du, V. Faber, and M. Gunzburger. Centroidal voronoi tessellations: applications and algorithms. SIAM Rev., Vol. 41, 6pp. 37-676, 1999.

[8] Q. Du, M. Gunzburger, L. Ju, and X. Wang. Centroidal Voronoi tessellation algorithms for image compression, segmentation, and multichannel restoration. J. Mathematical Imaging and Vision, Vol. 24, pp. 102-119, 2006.
[9] Q. Du, M. Gunzburger, and L. Ju. Advances in studies and applications of centroidal Voronoi tessellations. Numer. Math. Theo. Meth. Appl., Vol. 3, pp. 119-142, 2010.

[10] P. Felzenszwalb and D. Huttenlocher. Efficient graph-based image segmentation. IJCV, Vol. 59, pp. 167-181, 2004.

[11] L. Grady. Random walks for image segmentation. IEEE PAMI, Vol. 28, pp.1768-1783, 2006.

[12] J.A. Hartigan and M.A. Wong. A k-means clustering algorithm. J. the Royal Statistical Society. Series C, Vol. 28, pp. 100-108, 1979.

[13] C. Li, C. Xu, C. Gui, and M.D. Fox. Level set evolution without re-initialization: A new variational formulation. In IEEE CVPR, pp. 430-436, 2005.

[14] D. Magee, A. Bulpitt, and E. Berry. Level set methods for the $3 \mathrm{~d}$ segmentation of ct images of abdominal aortic aneurysms. in Proc. Medical Image Understanding and Analysis, pp. 141-144, 2001.

[15] D. Martin, C. Fowlkes, D. Tal, and J. Malik. A database of human segmented natural images and its application to evaluating segmentation algorithms and measuring ecological statistics. In IEEE ICCV, Vol. 2, pp. 416-423, 2001.

[16] F. Meyer. Topographic distance and watershed lines. Signal Processing, Vol. 38, pp. 113-125, 1994.

[17] R. Nock and F. Nielsen. Statistical region merging. IEEE PAMI, Vol. 26, pp. 1452-1458, 2004.

[18] A. Okabe, B. Boots, K. Sugihara, and S. Chiu. Spatial Tessellations: Concepts and Applications of Voronoi Diagrams. Wiley, Chichester, 2000.

[19] M.J. Powell. An efficient method for finding the minimum of a function of several variables without calculating derivatives. Computer Journal, Vol. 7, pp. 152-162, 1964.

[20] R.C. Reed. The Superalloys Fundamentals and Applications. Cambridge Press, 2001.

[21] J. Shi and J. Malik. Normalized cuts and image segmentation. IEEE PAMI, Vol. 22, pp. 888-905, 1997.

[22] D.N. Sparks. Euclidean cluster analysis. J. the Royal Statistical Society. Series C (Applied Statistics), Vol. 22, pp. 126130, 1973.

[23] H. Späth. Cluster Dissection and Analysis. Theory, FORTRAN Programs, Examples. Prentice Hall, Upper Saddle River, 1985.

[24] G.F. Vander Voort, F. J. Warmuth, S. M. Purdy, and A. Szirmae. Metallography: Past, Present, and Future (75th Anniversary Volume). ASTM,1993.

[25] F. Vivodtzev, L. Linsen, G.P. Bonneau, B. Hamann, K.I. Joy, and B.A. Olshausen. Hierarchical isosurface segmentation based on discrete curvature. In Proc. Conf. Data Visualization, pp. 249-258, ACM Press, 2003.

[26] J. Wang, L. Ju, and X. Wang. An edge-weighted centroidal voronoi tessellation model for image segmentation. In IEEE Trans. Image Processing, Vol. 18, pp. 1844-1858, 2009.

[27] R.T. Whitaker. A level-set approach to $3 \mathrm{~d}$ reconstruction from range data. IJCV, Vol. 29, pp. 203-231, 1998.

[28] P. Arbelaez, M. Maire, C. Fowlkes, and J. Malik. Contour Detection and Hierarchical Image Segmentation. IEEE PAMI, Vol. 33, pp. 898-916, 2011. 